\title{
Editorial note to: \\ G. F. R. Ellis, \\ Relativistic cosmology
}

\author{
Bill Stoeger
}

Published online: 29 January 2009

(C) Springer Science+Business Media, LLC 2009

Keywords Cosmology $\cdot$ Relativistic models · Golden Oldie

\section{Editor's note}

\section{By Bill Stoeger}

Few papers in relativistic cosmology have been as influential and as frequently cited as George Ellis' contribution to the 1969 Varenna Summer School. It is, therefore, very fitting that it should be republished and made more accessible as a "Golden Oldie" in General Relativity and Gravitation. Although it is primarily a synthesis of a number of earlier results, ${ }^{1}$ rather than a presentation of original work, it clearly and compellingly sets out a powerful general framework for describing and analyzing cosmological models. This is done in terms of splitting space-time into space + time by defining a projection tensor $h_{a b}$, related to the space-time metric $g_{a b}$ and to its fluid 4-velocity $u^{a}$ and using it to define and project geometric and kinematic quantities into the three-dimensional rest-spaces it defines with respect to $u^{a}$. The definition of the kinematical quantities themselves, the vorticity, shear, expansion and acceleration parameters, provides the basis for classifying important properties of cosmologies and for deriving the dynamical equations, like Raychaudhuri's equation, related to

\footnotetext{
1 These earlier results are primarily those of the Hamburg Group, particularly those of Ehlers and Trümper (see, for instance, the "golden oldie" of Ehlers [1], which is a translation of a much earlier paper [2], the 1967 paper of Trümper [3], and references therein) and of Hawking [4].
}

The republication of the original paper can be found via doi:10.1007/s10714-009-0760-7.

B. Stoeger $(\varangle)$

Vatican Observatory Group, Steward Observatory, The University of Arizona, Tucson, AZ, USA

e-mail: wstoeger@as.arizona.edu 
the Einstein field equations, the Ricci identities and the Bianchi identities, describing how these properties are sourced and how they in turn influence other features of space-times. All in all this framework constitutes a transparent, understandable and easily applied method for studying the characteristic features of any cosmology and quickly drawing general, qualitative and semi-quantitative conclusions about its evolution. The parallel Newtonian/General Relativity treatment Ellis employs in his exposition is an original contribution and unavailable elsewhere.

In particular, Ellis' Varenna Lectures popularized the importance and developed the applications of the Raychaudhuri equation, whose significance was first stressed by Ehlers, as well as the important role of the Weyl tensor in cosmology, its natural splitting into "electric" and "magnetic" components and the consequent "Maxwell form" of their Bianchi identities. This powerful and intuitively elegant framework has provided the key to understanding cosmological models and issues which go far beyond those confined to the Friedmann-Lemaittre-Robertson-Walker (FLRW) point of view, valuable as that has been in itself. Furthermore, Ellis has been careful to specialize his treatment to FLRW cases throughout the paper, which has led to a deeper appreciation of these standard models and their connections with more general ones.

Within this overall framework Ellis also presents a careful covariant geometric discussion of observations in cosmology, including the simple generic expressions for the redshift, the observer area and luminosity distances, the null-shear parameter, and galaxy number counts, again specializing the results to spherically symmetric models, including the important FLRW cases. A key original contribution in the treatment is clear geometric proof of the reciprocity theorem ${ }^{2}$ — essentially that the observer area distance $r_{o}$, which is equivalent to the angular diameter distance, and the galaxy area distance $r_{g}$ have the simple relationship $r_{g}^{2}=r_{o}^{2}(1+z)^{2}$ in any space-time, no matter how inhomogeneous. Here $z$ is the redshift. Connected with this, of course, is that all the observable distances, including luminosity distance, one can define are generically related to one another by factors of $(1+z)$. These relationships do not depend on the space-time being FLRW.

Finally, Ellis' Varenna Lectures contain several other nice features. They include a simple and transparent treatment of the matter content of the universe, and of perfect-fluid equations of state, along with a very helpful thermal history of the universe. Near the end of the paper, there is a brief but helpful discussion of the implications of the isotropy of the cosmic microwave background radiation (CMWBR), referring to the Ehlers, Geren and Sachs theorem [6], and giving limits on large-scale anisotropies and inhomogeneities, particularly the vorticity and the shear. There follows a brief but rather complete sketch of the important early work on the existence and nature of singularities and horizons, and their presence in various cosmological models. What the paper does not include are references to the inflationary universe idea, and discussions of the evidence for and nature of dark matter and dark energy_issues that have come up since the paper was completed. Any updated version of the paper would surely discuss these important topics.

\footnotetext{
2 See the 2007 Golden Oldie of Etherington's paper [5] on this theorem.
} 
The impact and influence of this paper and the methods it describes has been pervasive throughout mathematical cosmology. Among the most important applications of the approach Ellis, following the Hamburg School, has promoted in his Varenna Lectures, as well as in his later Cargèse Lectures [7], has been the $3+1$ covariant and gauge-invariant perturbation program initiated by Ellis and Bruni $[8,9]$ for dust (pressure $p=0$ ) and perfect-fluid FLRW cosmologies. This program has been generalized by Dunsby, Bruni and others [10-12] to a large variety of cases: perfect fluids, imperfect fluids, universes with multiple matter components and different 4velocities, kinetic theory, the Einstein static universe and even higher dimensional theories. This last topic has been extensively pursued by Roy Maartens and others, and is one of the outstanding applications of these $3+1$ methods $[13,14]$.

Probably the most far reaching and significant application has been by Anthony Lasenby, Anthony Challinor and Anthony Lewis applying the techniques to kinetic theory and using it to describe CMWBR anisotropies [15-18]. This led to the CAMB computer programme and its descendents, developed by the Cavendish group at Cambridge. These give detailed CMWBR anisotropy and polarization results for all spatial curvatures. Another closely related application of the methods was the generalization of the Ehlers-Geren-Sachs theorem by Stoeger et al. [19] to almost-isotropic, radiation-plus-dust cases, using the related extended formalism of Ellis, Matravers and Treciokas [20]. This led to using the CMWBR to place limits on the various types of anisotropies and inhomogeneities in the universe, like shear, vorticity and large-scale density gradients [21-23].

Other significant programs strongly influenced by this paper are the development of gauge-invariant higher order perturbation theory by Clarkson and others [24], investigations of exact cosmological solutions, such as those by John Wainwright and his group exploring Bianchi universes, ${ }^{3}$ the research on gravitational waves by Peter Hogan, George Ellis, and Emir O'Shea [31-33], the investigations of magnetic fields and gravitational waves in cosmology by Peter Dunsby and his collaborators [34-36], the work on the Newtonian limit in cosmology by Henk van Elst and George Ellis $[37,38]$, and the work on integrability conditions for cosmologies which are special cases (e. g., when $E=0, H=0$ ("silent universes"), $\nabla \cdot E=0, \nabla \cdot H=0$, etc.) [39]. This last topic has recently been revisited and developed by Norbert Van den Bergh and Lode Wylleman and their group [40,41].

Thus, the approaches and techniques developed and promoted by Ellis' Varenna Lectures have become essential tools for theoretical cosmology, and have been very fruitful in pushing the field forward in a variety of important ways. There is every indication that they shall continue to be employed frequently with profit. It is, therefore, a distinct privilege to present this reprinting of George Ellis' "Relativistic Cosmology" as a "Golden Oldie" in General Relativity and Gravitation.

Acknowledgment I am very grateful for George Ellis's advice and input in writing this introduction.

\footnotetext{
3 See the volume edited by Wainwright and Ellis [25], especially the contributions of Ellis and Wainwright and their collaborators, and that of Dunsby, i.e. Chapters 1-3, 6-9, 12, 14-15. See also: Nilsson et al. [26], Hewitt et al. [27], van Elst et al. [28], Hewitt et al. [29], Lim et al. [30], and references therein.
} 


\section{George Ellis: a brief autobiography}

\section{By George Ellis}

George Ellis studied mathematics and physics at the University of Cape Town, graduating in 1961, and completed his Ph.D. degree in the Department of Applied Mathematics and Theoretical Physics ("DAMTP") at Cambridge University under the supervision of Dennis Sciama in 1964. He was first a post doc and then a Lecturer at Cambridge, having the fortune to work with many talented people, including Dennis Sciama, John Stewart, Malcolm MacCallum, Andrew King, Bob Treciokas, and Stephen Hawking on a number of papers on relativity and cosmology; he co-authored the book The Large Scale Structure of Space Time with Stephen Hawking, which appeared in 1973.

He became Professor of Applied Mathematics at the University of Cape Town in 1974, setting up a relativity research group and writing many papers and various books on relativity and cosmology. His Ph.D. Students here included Stanley Nel, Roy Maartens, and Mark Madsen. He also looked at many other issues, including housing policy, quality of life measurement, and science policy, and then latterly issues in complexity, the nature of the brain, and the philosophy of cosmology. Over the years he has been visiting professor at the universities of Texas, Hamburg, Chicago, Alberta, Boston University, and London University (Queen Mary), the Max Planck Institute of Astrophysics (Garching), the Vatican Observatory (Castel Gandolfo), and Professor of Cosmic Physics at SISSA, Trieste where he was also a lecturer at ICTP. He won the Gravity Research Foundation Essay Competition 1st Prize in 1979. He has been President of the International Society of General Relativity and Gravitation ("GRG") and of the Royal Society of South Africa; he was a founding member of the Academy of Science of South Africa, which he represented on the Inter-Academy Council (IAC), Amsterdam.

He is currently co-Editor in Chief of the GRG Journal (with Hermann Nicolai). His books include "Low Income Housing Policy in South Africa", with D Dewar (Urban Problems Research Unit, University of Cape Town, 1979), "Flat and Curved SpaceTimes" with R M Williams (Oxford University Press, 1988, 2000), "The Renaissance of General Relativity and Cosmology”, with A Lanza and J Miller (University Press, Cambridge, 1993), "Science Research Policy in South Africa" (Royal Society of South Africa, 1994), "On The Moral Nature of the Universe: Cosmology, Theology, and Ethics", with N Murphy (Fortress Press, 1996), "Is The Universe Open or Closed? The Density of Matter in the Universe", with P Coles (Cambridge University Press, 1997), "The dynamical systems approach to cosmology", with J Wainwright (Cambridge University Press, 1996), "The Far Future Universe" (Templeton Foundation Press, 2002), and "The universe around us: an integrative view of science and cosmology", an e-book, available at http://www.mth.uct.ac.za/ ellis/cos0.html.

His webpage is at http://www.mth.uct.ac.za/ ellis/.

Editor's comment: A more detailed biography of George Ellis, by Malcolm MacCallum, Roy Maartens and Peter Dunsby, together with a 20-page bibliography of his papers, appeared in Gen. Relativ. Gravit. 32, pp 977-979 (2000). 


\section{References}

1. Ehlers, J.: Gen. Relativ. Gravit. 25, 1225 (1993)

2. Ehlers, J.: Abh. Akad. Wiss. und Lit. Mainz, Math. Nat. K1., Nr. 11, 792-837 (1961)

3. Trümper, M.: Zeits. F. Astrophys. 66, 215 (1967)

4. Hawking, S.W.: Astrophys. J. 145, 544 (1966)

5. Etherington, I.M.H.: Gen. Relativ. Gravit. 39, 1047 (2007) [originally published: Philosophical Magazine, series 7 15, Nr. 18, 761 (1933)]

6. Ehlers, J., Geren, P., Sachs, R.K.: J. Math. Phys. 9, 1344 (1968)

7. Ellis, G.F.R.: In: Schatzmann, E. (ed.) Cargèse Lectures in Physics. Gordon and Breach, New York (1973) — which also contains the $3+1$ covariant decomposition of Maxwell's equations

8. Ellis, G.F.R., Bruni, M.: Phys. Rev. D 40, 1804 (1989)

9. Ellis, G.F.R., Hwang, J.-C., Bruni, M.: Phys. Rev. D 40, 1804 (1989); 40, 1819 (1989)

10. Dunsby, P.K.S.: Class. Quantum Grav. 8, 1785 (1991)

11. Bruni, M., Dunsby, P.K.S., Ellis, G.F.R.: Astrophys. J. 395, 34 (1992)

12. Dunsby, P.K.S., Bruni, M., Ellis, G.F.R.: Astrophys. J. 395, 54 (1992)

13. Maartens, R.: Phys. Rev. D 62, 084023 (2000)

14. Maartens, R.: Living reviews in relativity. http://www.livingreviews.org/lrr-2004-7

15. Challinor, A.D., Lasenby, A.N.: In: Sanchez N, Zichichi A (eds.) Current Topics in Astrofundamental Physics: Primordial Cosmology, pp. 409ff (arXiv: astro-ph/9711028v3). Kluwer, Dordrecht (1998)

16. Challinor, A.D., Lasenby, A.N.: Phys. Rev. D 58, 023001 (1998)

17. Challinor, A.D., Lasenby, A.N.: Astrophys J. 513, 1 (1999)

18. Lewis, A.N., Challinor, A.D., Lasenby, A.N.: Astrophys. J. 538, 473 (2000)

19. Stoeger, W.R., Maartens, R., Ellis, G.F.R.: Astrophys. J. 443, 1 (1995)

20. Ellis, G.F.R., Matravers, D.M., Treciokas, R.: Ann. Phys. (N. Y.) 150, 455 (1983)

21. Maartens, R., Ellis, G.F.R., Stoeger, W.R.: Phys. Rev. D 51, 1525 (1995)

22. Maartens, R., Ellis, G.F.R., Stoeger, W.R.: Phys. Rev. D 51, 5942 (1995)

23. Stoeger, W.R., Araujo, M.E., Gebbie, T.: Astrophys. J. 476, 435 (1997)

24. Clarkson, C.: Phys. Rev. D 70, 103524 (2004)

25. Wainwright, J., Ellis, G.F.R. (eds.): Dynamical Systems in Cosmology, 317pp. Cambridge University Press, Cambridge (1997)

26. Nilsson, U.S., Hancock, M.J., Wainwright, J.: Class. Quantum Grav. 17, 3119 (2000)

27. Hewlett, C.G., Bridson, R., Wainwright, J.: Gen. Relativ. Gravit. 33, 65 (2001)

28. van Elst, H., Uggla, C., Wainwright, J.: Class. Quantum Grav. 19, 51 (2001)

29. Hewlett, C.G., Horwood, J.T., Wainwright, J.: Class. Quantum Grav. 20, 1743 (2003)

30. Lim, W.C., Uggla, C., Wainwright, J.: Class. Quantum Grav. 23, 2607 (2006)

31. Hogan, P., Ellis, G.F.R.: Class. Quantum Grav. 14, A171 (1997)

32. Hogan, P., O'Shea, E.M.: Phys. Rev. D 65, 124017 (2002)

33. O'Shea, E.M.: Phys. Rev. D 69, 064038 (2004)

34. Tsagas, C.G., Dunsby, P.K.S., Marklund, M.: Phys. Lett. B 561, 17 (2003)

35. Betschart, G., Zunckel, C., Dunsby, P.K.S., Marklund, M.: Phys. Rev. D 72, 123514 (2005)

36. Zunckel, C., Betschart, G., Dunsby, P.K.S., Marklund, M.: Phys. Rev. D 73, 103509 (2006)

37. van Elst, H., Ellis, G.F.R.: Class. Quantum Grav. 15, 3545 (1998)

38. Hwang, J.-C., Noh, H.: Gen. Relativ. Gravit. 38, 703 (2006)

39. van Elst, H., Uggla, C., Lesame, W.M., Ellis, G.F.R.: Class. Quantum Grav. 14, 1141 (1997)

40. Van den Bergh, N., Wylleman, L.: Class. Quantum Grav. 21, 2291 (2004)

41. Wylleman, L., Van den Bergh, N.: Class. Quantum Grav. 23, 329 (2006) 